\title{
LOS HUIPILES COMO RECURSO DIDÁCTICO EN LA ENSEÑANZA DE LA GEOMETRÍA
}

\section{HUIPILES AS A TEACHING RESOURCE IN THE TEACHING OF GEOMETRY}

\section{Referencia del artículo}

Pérez, V. (2019). Los huipiles como recurso didáctico en la enseñanza de la geometría. Revista Científica del SEP. 2(1), 1-9. DOI: https://doi.org/10.36958/sep.v2i01.11

\author{
Vilma Isabel Pérez Hernández \\ isabelp11629@gmail.com \\ Licenciada en Educación de la matemática y la física \\ Centro Universitario de Occidente \\ Universidad de San Carlos de Guatemala
}

\author{
Carlos Amilcar Fuentes Fuentes \\ caffuentes7@gmail.com \\ Maestro en Ciencias en Matemática Educativa \\ Centro Universitario de Occidente \\ Universidad de San Carlos de Guatemala
}

Fecha de recepción: 12/08/2019

Fecha de aceptación: 25/10/2019

\section{RESUMEN}

Se reporta un trabajo de investigación que busco responder la pregunta ¿Cuáles son los resultados de utilizar los huipiles como recurso didáctico en la clase de Geometría? El método se basó en el diseño y aplicaciónde una secuencia didáctica, que integra fotografías de los trajes de las mujeres de Santa Catarina Ixtahuacán, Sololá, departamento del Altiplano de Guatemala, como recurso didáctico para la enseñanza de la Geometría Euclidiana.

Los temas abordados en las sesiones de clase fueron propiedades y relaciones geométricas con respecto a los lados, ángulos y diagonales de las formas, cálculo de área y perímetro de figuras planas, dirigido a estudiantes del ciclo de educación básica.

La secuencia didáctica se organizó en tres momentos: a) Introducción y diagnóstico, b) Desarrollo de contenidos, y c) Evaluación final.

De los resultados obtenidos, se puede mencionar que los estudiantes alcanzaron un nivel alto de participación, espontaneidad, atención, motivación, trabajo en equipo, desarrollo de liderazgo, manejo de propiedades y relaciones entre los lados, ángulos y diagonales de las figuras, además de la profundización en el uso de estrategias para calcular área y perímetro de figuras planas.

\section{PALABRAS CLAVE}

Secuencia didáctica, güipil, geometría euclidiana, fotografía, recurso didáctico

\section{ABSTRACT}

We report a research work that sought to answer the question: What are the results of using traditional costumes as a teaching resource in the Geometry class? The method was based on the design and application of a didactic sequence, which integrates photographs of the costumes of the women of Santa Catarina Ixtahuacán, Sololá, Department of the Altiplano of Guatemala, as a teaching resource for the teaching of Euclidean Geometry.

The topics addressed in the class sessions was: properties and geometric relationships with respect to the sides, angles and diagonals of the forms, calculation of area and perimeter of flat figures, with at students of the basic education cycle.

The didactic sequence was organized in three moments: a) Introduction and diagnosis, b) Content development, and c) Final evaluation.

From the results obtained, it can be mentioned that the students reached a high level of participation, spontaneity, attention, motivation, team work, leadership development, property management and relationships between the sides, angles and diagonals of the figures. They deeped in the use of strategies to calculate area and perimeter of the flat figures.

\section{KEYWORDS}

Didactic sequence, regional costume, euclidean geometry, photography, didactic resource 


\section{INTRODUCCIÓN}

Se presentan los resultados del estudio titulado "Los huipiles como recurso didáctico en la enseñanza de la Geometría", como una propuesta para el estudio de propiedades, cálculo de áreas y perímetros de figuras geométricas planas. Para aclarar, un huipil es una prenda femenina que las mujeres mayas visten en la parte del tronco, cubriendo desde el cuello hasta la cadera o medio muslo, es de manga corta y está adornada con vistosos bordados de figuras o tejidos a mano, que expresan la cosmovisión del pueblo maya.

Con este estudio se pretende contribuir a minimizar las dificultades que, según (González 2014), presentan los estudiantes en el aprendizaje de la Geometría, en el cálculo de áreas y perímetros cuya enseñanza, según (Mántica, del Maso Götte \& Marzioni 2002), se reduce a la aplicación y memorización de fórmulas en ejemplos idénticos a los que el profesor resuelve.

En el estudio realizado se diseñó una secuencia didáctica con actividades que integraron fotografías de los trajes de la región de occidente de Guatemala; previo a ello se obtuvo información del recurso con tejedoras de Santa Catarina Ixtahuacán y posteriormente se realizó una sesión fotográfica de los distintos trajes que utilizan las mujeres, de acuerdo a la ocasión.

La secuencia inicia con el descubrimiento de formas geométricas presentes en los diseños de los huipiles, los estudiantes realizaron mediciones y trazos de las figuras, logrando establecer relaciones entre los lados, ángulos y diagonales de las mismas y propusieron estrategias para calcular el perímetro y área de las formas; fue necesario recordar las definiciones de paralelismo y perpendicularidad, para la comprensión de las propiedades de las figuras geométricas planas.

De los resultados obtenidos sobre la utilización de fotografías de los trajes regionales en la secuencia didáctica se pueden mencionar: el interés y motivación por descubrir la geometría en el entorno, debido a la conexión del conocimiento geométrico con las producciones culturales de las comunidades.

La exploración y manipulación de materiales orientaron al estudio de las componentes de las figuras, relaciones, propiedades geométricas y manejo de estrategias para la medición de superficies planas. Las reflexiones realizadas por el estudiante contribuyeron a construir o reconstruir conceptos geométricos de las figuras planas.

\section{PLANTEAMIENTO DEL PROBLEMA}

En un informe que presenta (MINEDUC 2006) con respecto a las pruebas realizadas a estudiantes de Tercero Básico del ciclo escolar 2013 del departamento de Quetzaltenango, se muestra un resultado satisfactorio del $18.55 \%$ en el área de Matemática, lo que indica que el $81.5 \%$ tienen dificultades en la comprensión de conceptos aritméticos, geométricos, algebraicos y estadísticos; en el informe se verifica, que en una prueba de 40 ítems el $17.5 \%$ evalúa el componente de geometría; dichos resultados requieren atención y propuestas para mejorar las dificultades que se presentan en el aprendizaje de conceptos geométricos.

Según (González 2014), y de acuerdo a la experiencia docente de los autores, los estudiantes confunden el concepto de área con el de perímetro, tienen la idea errónea de que el área es el número de unidades que recubren una superficie y existe una probabilidad alta de que reprueben exámenes escritos porque no realizan procedimientos correctos.

¿Y qué sucede en la mayoría de instituciones educativas?, según (Vargas 2013) la enseñanza de la geometría se realiza de una manera tradicional, el discurso del profesor se considera el principal medio didáctico, sin considerar otros recursos que facilitan la comprensión de los conceptos geométricos; y para calcular el área y perímetro de figuras planas, según (Mántica, et. al. 2002), se limitan a la aplicación y memorización de fórmulas ocasionando dificultad en la comprensión del valor que representa las unidades cuadradas que cubren la superficie de una figura plana, además, según (Marmolejo \& Gonzáles 2015), los estudiantes presentan dificultades en la resolución de problemas abstractos.

Otro factor que ocasiona dificultades en la comprensión de los conceptos de geometría es la falta de visualización a través de un medio concreto, por ejemplo, la medición de la superficie de una figura a través de la cuadrícula, según (Marmolejo \& Gonzáles 2015), establece una relación entre ella y la unidad de medida a utilizarse.

Las acciones de cortar, mover, pegar y trasformar una figura en otra, son fundamentales para la comprensión de la medida de superficie. Otros estudios indican que el uso de herramientas culturales facilita el trabajo de medición de perímetro y área de figuras planas según (Bronzina, Chemello \& Agrasar 2009), porque el estudiante se enfrenta a situaciones cotidianas.

Por tanto, partiendo de la problemática expuesta anteriormente y tomando como base la experiencia docente y queriendo ofrecer al estudiante todas las posibilidades para el desarrollo de habilidades y niveles de razonamiento geométrico, se planteó la siguiente pregunta: 
¿Cuáles son los resultados de utilizar los huipiles como recurso didáctico en la clase de la geometría?

\section{MARCO CONCEPTUAL}

La geometría se observa en la naturaleza, objetos y expresiones culturales, y si el aprendizaje de dicho objeto matemático se asocia a las diferentes manifestaciones de la sociedad como el arte, diseños arquitectónicos, la naturaleza adquiere significado y se convierte en una gran fuente de experiencia para el estudiante.

(Crespo 2005), expone que a través de la geometría se desarrollan habilidades y capacidades para juzgar y valorar objetivamente la funcionalidad y simbología del conocimiento matemático; además permite comprender, describir e interactuar con el espacio en el que se vive, observar el mundo de manera geométrica en contextos y situaciones, a través de la percepción visual y representación de objetos en dos o tres dimensiones, acciones que, según (MEN2004), permitirá la construcción del pensamiento Geométrico.

Según (Morales s.f.) las manifestaciones culturales como los tejidos proporcionan una gama de mosaicos con formas geométricas que combinan formas poligonales en sus diseños; el estudio de la geometría en los diseños de los tejidos mayas, se convierte en una herramienta para la enseñanza de dicho conocimiento matemático; por ejemplo, según (Morales s.f.) y (Godino \&Ruíz2002), en los huipiles guatemaltecos se observan símbolos con forma de rombos o romboides, triángulos ordenados en filas, cadenas en forma horizontal o diagonal, como se observan en las figuras1 y 2 .

Figura 1

Huipil moderno de Santa Catarina Ixtahuacán.

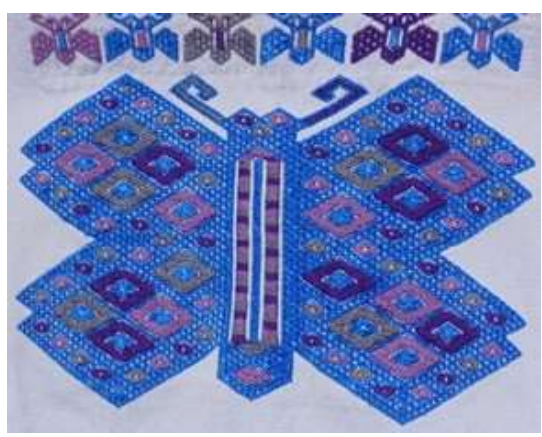

Fotografía: Carlos Fuentes
Figura 2

Huipil ceremonial de Santa Catarina Ixtahuacán

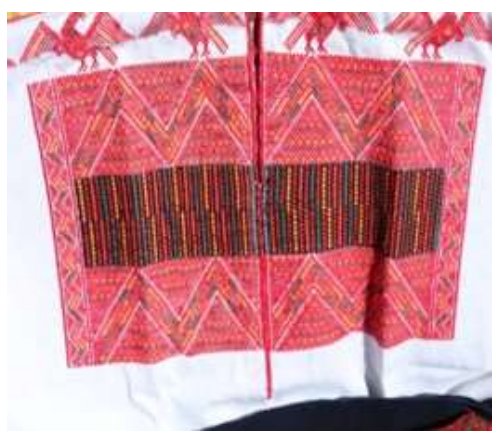

Fotografía: Pedro Pérez

Observando las anteriores fotografías, es evidente que la geometría es vista como una práctica social porque forma parte de las actividades de cada grupo de la sociedad; también se considera como una teoría formal entre el pensamiento espacial y pensamiento métrico.

Según (Casasbuenas, Cifuentes, \& Ortega de N., 1998, pág. 62), la geometría tiene como propósito "definir, justificar, deducir y comprender algunas demostraciones" de los objetos y fenómenos que rodean a las personas. La geometría es una rama de las matemáticas que permite la abstracción, generalización, definición y deducción formal a partir de axiomas, que trata de explicar utilizando conceptos de geometría situaciones, fenómenos y hechos reales.

Debido a los diseños geométricos de los huipiles de las mujeres mayas del altiplano del país, las figuras que se consideraron para este estudio fueron: cuadrado, rectángulo, rombo, triángulo, trapecio y algunos polígonos regulares como el pentágono y hexágono, que son figuras a estudiar según el currículo nacional base de Guatemala.

Se realimentaron conceptos de perímetro y área, para lo cual se consideró la definición de (Peralta \& Murillo 2009), que indica serla medida del contorno de la superficie y que puede determinarse mediante la suma de las longitudes de los lados de un polígono.

El área puede definirse como la medida de una superficie delimitada por un determinado perímetro cuya medida o patrón estándar es el metro cuadrado con sus múltiplos y submúltiplos.

Las unidades de medida empleadas en la secuencia didáctica fueron las correspondientes al sistema métrico decimal, es decir, centímetro $(\mathrm{cm})$, decímetro $(\mathrm{dm})$, metro $(\mathrm{m})$. 
En cuanto a los medios u objetos necesarios para ayudar a construir, entender y reforzar conceptos y procedimientos, (Villarroel \& Sgreccia, 2011) les llama materiales didácticos concretos, convirtiéndose en recursos que el estudiante y profesor utilizan en el proceso de aprendizaje y enseñanza de la geometría, para lograr el tránsito en los diferentes niveles de razonamiento geométrico.

De los materiales didácticos más comunes que se utilizan para el desarrollo de habilidades y adquisición de conocimiento geométrico se puede mencionar: el geoplano, mecano, papel doblado (Origami o Papiroflexia), tangram, espejos, software de geometría, modelos fijos en dos y tres dimensiones, rompecabezas geométricos y objetos del entorno natural, artificial y artístico representados por medio de la fotografía. El uso de la fotografía como material didáctico, es relativamente nuevo y es un recurso que permite la exploración de conocimientos y estudio de la percepción e interpretación de las matemáticas, desarrolla la capacidad de observación y estimula la creatividad, obligando a reflexionar e interpretar la realidad y desde luego, contribuyendo a la construcción del conocimiento geométrico.

El recurso fotográfico captura imágenes con nociones matemáticas, para visualizar formas y propiedades geométricas, graficas, procesos de crecimiento, entre otros (Flores, Lupiañez, Berenguer, Marín, \& Molina , 2011).

Existen varios dispositivos en la actualidad que pueden ser utilizados en el aula, por ejemplo: las cámaras fotográficas, los teléfonos y las tablets, y según (Fuentes 2017) son un excelente recurso didáctico para el aprendizaje de la geometría.

Los materiales didácticos pueden facilitar el estudio del objeto matemático en una secuencia didáctica, que (DiazBarriga2013) define como la organización de las actividades de aprendizaje que se desarrollarán con estudiantes para alcanzar los objetivos propuestos y las situaciones de aprendizaje que son significativas y facilitan la construcción del conocimiento.

Sobre el papel del docente, según (Chacón, Trejos, Vargas, \& Venegas , 2005), será un mediador entre conocimiento y estudiante y el responsable de la selección o creación de actividades, para el descubrimiento o deducción de conceptos y teoremas matemáticos.

Sobre la organización adecuada y las actividades de aprendizaje con objetos concretos, (Roldán \& Réndon 2014), argumentan que favorece la construcción y desarrollo del pensamiento geométrico, que cambia la forma tradicional de exponer la teoría euclidiana, ya que permite la práctica del conocimiento matemático y la adquisición de un aprendizaje significativo. Diaz \& Escobar (2006), también apoya el uso del material concreto, puespermite la identificación de caras, formas, relaciones, aristas, direcciones y vértices de las formas geométricas, que finalmente permite el paso al plano abstracto.

Sobre la secuencia didáctica aplicada en este estudio, se organizó de acuerdo a la estructura de las situaciones didácticas de Brousseau, definiéndose según (Salinas 2010), como un tipo de interacción entre el estudiante y su entorno, docente, escuela y sistema educativo en general.

En la situación didáctica el docente organiza la relación entre estudiante y medio, y crea un ambiente que permite la manifestación $y / o$ construcción del conocimiento, según (D’Amore 2008), a través del trabajo autónomo y la resolución de problemas, para ello se requiere considerar las necesidades, intereses y situaciones que lo involucra.

En (Salinas 2010), las Situaciones Didácticas se dividen en:

a. Situación de Acción: proceso ordenado que realizael estudiante en su aprendizaje para adquirir métodos de resolución de problemas, el docente orienta e interviene provocando la problematizacióna fin de guiar la situación.

b. Situación de Formulación: desarrollo de la capacidad de reconocimiento de figuras geométricas, según (Peralta y Murillo2009), identificación de propiedades, descomposición y reconstrucción de figuras y manejo de un sistema lingüístico través de la explicación y análisis realizado del saber adquirido.

c. Situación de Validación: organiza y presenta enunciados en demostraciones, construyen conceptos de nuevos saberes para validar argumentos y razonamientos lógicos expresados en la situación de formulación, el docente guía la situación a través de interrogantes.

d. Situación de Institucionalización: proceso metadidáctico que surge de la reflexión del docente, toma como base los procesos generados por el estudiante, sobre la búsqueda de un conocimiento específico.

La vinculación de figuras, cuerpos geométricos concretos y 
la construcción de definiciones matemáticas a través de la comprobación y demostración de resultados permiten la construcción del pensamiento geométrico, según (Diaz y Escobar2006, p 27)el pensamiento espacial o geométrico es el "Conjunto de procesos cognitivos mediante los cuales se construyen y se manipulan las representaciones mentales de los objetos del espacio, las relaciones entre ellos, sus transformaciones y sus diversas traducciones a representaciones materiales", es decir, la interacción del sujeto con objetos que se encuentran en el espacio, los procesos de representación, manipulación y creación de conceptos mentales dan surgimiento al pensamiento geométrico.

Según (Casasbuenas, Cifuentes \& Ortega1998), el pensamiento geométrico se desarrolla en tres momentos:

i. Las relaciones que se establecen entre los objetos que se encuentran en el espacio y la ubicación y relación del sujeto con respecto a estos objetos y el espacio.

ii. La necesidad de medición de esta relación, que tan cerca o lejos está el objeto y sujeto con respecto al espacio.

iii. El estudio de las propiedades espaciales, que involucran su relación y medición.

A través del pensamiento espacial se construye, maneja, transforma y utiliza los sistemas geométricos, sistemas que están formados por: puntos, líneas rectas y curvas, regiones planas o curvas, cuerpos sólidos, transformaciones y relaciones espaciales. Según (Díaz \& Escobar 2006) el estudio de la geometría es una alternativa para explorar y representar el entorno por medio del espacio abstracto.

El estudio del espacio y movimiento se interrelaciona con el componente de formas patrones y relaciones del currículo nacional base de Guatemala, partiendo de la ejecución del currículo y la experiencia docente, requiere de un ambiente donde el estudiante pueda manipular, dibujar, calcular, construir y producir modelos mentales y operatorios, a través de distintas actividades y recursos que generen un aprendizaje significativo.

\section{METOdOLOGÍA}

Para tener una visión general del proceso de tejido de los huipiles se decidió visitar el municipio de Santa Catarina Ixtahuacán, Sololá, ubicado en el altiplano de Guatemala, para realizar una entrevista con la Licda. Isabel Reina Tepaz Raxuleu, tejedora de dicho lugar, de quién se obtuvo información sobre los diseños de los trajes que utilizan las mujeres; diversidad de trajes que visten de acuerdo a la ocasión, edad, y estatus social.

Entre los distintos trajes mencionados por la entrevistada, se encuentra el traje de diario, de gala, el ceremonial, el petate y los modernos que tienen figuras no tradicionales.

En la visita a la entrevistada, se tomaron fotografías de distintas prendas de mujeres; de todas ellas, se seleccionaron 15 para imprimir en tamaño de , para formar parte del material didáctico a utilizar con el grupo de estudiantes.

Las fotografías se forraron con una película plástica, para manipularlas sin ningún riesgo de deterioro. La decisión de usar fotografías en lugar de los trajes típicos en físico, obedece al alto costo y delicadeza de los mismos, así como a la cantidad de estudiantes con quienes se debía desarrollar la secuencia didáctica y por facilidad en la manipulación.

El diseño y planificación de la secuencia didáctica se dividió en tres momentos: introducción y diagnóstico, desarrollo de contenidos y evaluación final.

Previo a la puesta en escena de la secuencia didáctica, se probó con un grupo de estudiantes del tercer grado del ciclo básico del nivel medio, acción que permitió evaluar, reorientar, corregir y mejorar el desarrollo de las actividades en la secuencia didáctica.

El estudio se realizó con un grupo de estudiantes de segundo básico, del Instituto Nacional Experimental "Dr. Werner Ovalle López" de la ciudad de Quetzaltenango, pues en dicho grado es donde, según el currículo nacional base, se deben abordar los temas contenidos en la secuencia didáctica. Los detalles de la aplicación definitiva de la secuencia didáctica se presentan a continuación:

\subsection{Introducción y diagnóstico}

Con una duración de 2 períodos de 35 minutos cada uno, para lo cual se organizaron a los estudiantes en grupos de seis integrantes, entregándoles una fotografía para el reconocimiento de figuras geométricas planas en los diseños presentes de los trajes y una guía de preguntas acerca de los conocimientos previos que poseen sobre las propiedades y cálculo de medidas de área y perímetros. Al final de la sesión, los estudiantes expusieron los resultados observados en la fotografía proporcionada, guiándose del cuestionario que respondieron. 


\subsection{Desarrollo de contenidos}

Etapa donde se propició la construcción y reconstrucción de conceptos. Esta etapa tuvo una duración de 10 períodos, proceso que consistió en trazo, medición y recorte de las formas geométricas planas en hojas milimetradas, utilizando instrumentos de medición. El orden en que se presentaron las figuras estudiadas permitió iniciar con el cuadrado, por tener las dimensiones de sus cuatro lados iguales y por la facilidad en la medición; luego se estudió el rectángulo, el rombo, el trapecio, el triángulo, el circulo, el pentágono y el hexágono.

También se estudió con el grupo la transformación de figuras como el rectángulo en paralelogramo, en este momento se definieron conceptos de paralelismo y perpendicularidad, para la comprensión de las propiedades de las figuras geométricas.

\subsection{Evaluación final}

con una duración de 2 períodos, desarrollada de la misma forma que el primer momento. Proceso que consistió en el reconocimiento de figuras planas, propiedades y medición de perímetros y áreas en fotografías distintas a las que se presentaron en la etapa de introducción y diagnóstico, pero siempre basándose en diseños de los trajes regionales, como se observa en las figuras 3 y 4 .

Figura 3

Identificación de triángulos y rectángulos

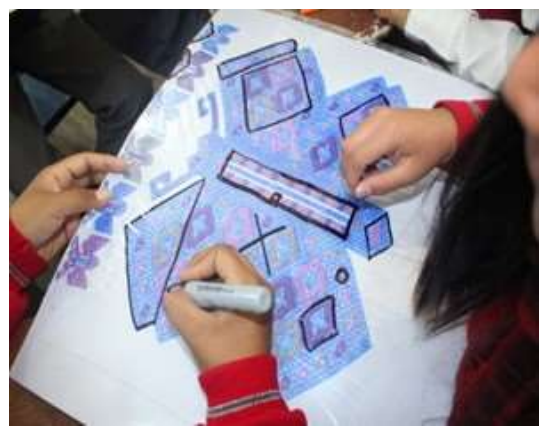

Figura 4

Construcción de un rombo

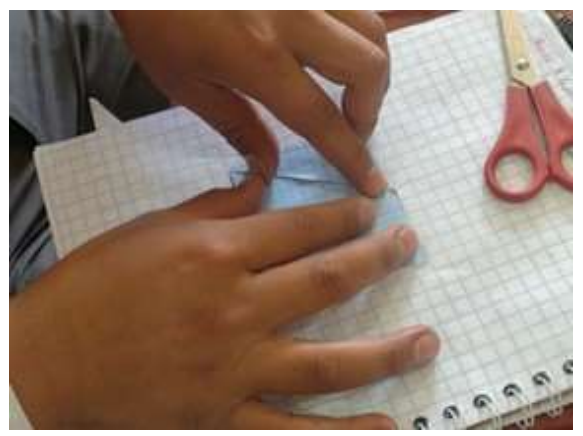

\section{RESULTADOS}

Recordando la pregunta que originó el presente estudio, ¿Cuáles son los resultados de utilizar los huipiles como recurso didáctico en la clase de la geometría?, se pueden presentar los siguientes resultados sobresalientes.

En la etapa de introducción y prueba diagnóstica, los estudiantes descubrieron formas geométricas en los diseños de los tejidos, señalaron líneas rectas y curvas, realizaron mediciones de lados y ángulos de las figuras, establecieron una conexión del conocimiento geométrico con producciones y concepciones de la cultura maya K'iche'; también se obtuvo una participación activa y en todo momento que se pudo captar la atención, el interés y trabajo en equipo durante el desarrollo de la prueba. En la exposición grupal se pudo apreciaren un nivel básico, el uso de vocabulario técnico sobre los conceptos geométricos y el análisis con ciertas limitaciones.

En la etapa de desarrollo de contenidos se estudiaron las figuras a través de la experimentación que consistió en una sesión de medición, trazo y transformaciones de las figuras geométricas; logrando como resultado, el descubrimiento de las relaciones entre los lados, ángulos y diagonales de los cuadriláteros, llegando a conclusiones tan importantes por parte de los estudiantes como: a) el rectángulo es un paralelogramo con ángulos rectos, b) el rombo es un paralelogramo con cuatro lados iguales. Además de las conclusiones mencionadas, los estudiantes usaron estrategias como el conteo de unidades cuadradas que forman la figura o transformaciones de unas figuras en otras, por ejemplo, cuadriláteros en romboides o rectángulos, para calcular el perímetro y área de las formas geométricas.

En la etapa de evaluación final describieron las propiedades generales de las figuras geométricas señaladas en las fotografías, clasificaron como cuadriláteros a las figuras observadas con forma de rectángulo, cuadrado, rombo; calcularon el área y perímetro de cada figura, por medio de procedimientos matemáticos, y las medidas de las figuras obtenidas fueron diferentes para cada grupo tomando en cuenta que las fotografías entregadas eran distintas.

\section{DISCUSIÓN DE RESULTADOS}

De acuerdo a los resultados de este estudio, la utilización de las fotografías de los diseños de los huipiles de las mujeres mayas de Santa Catarina Ixtahuacán, permitió la participación espontánea del estudiante, haciendo de la secuencia didáctica una propuesta novedosa que puede implementarse en los 
salones de clase, para abordar temas de geometría, pues proporciona información sobre el proceso cognitivo que realiza en la comprensión y aprendizaje de la geometría.

El recurso genera información relacionada al conocimiento matemático, cuyo estudio motiva y orienta el proceso de aprendizaje en una actividad fácil, interesante, experimental y práctica para el estudiante y propicia un ambiente de confianza. Es una propuesta que cambia la forma magistral, que en ocasiones se usa para abordar la enseñanza de la geometría, la cual puede limitar el desarrollo del pensamiento geométrico y la construcción o reconstrucción de conceptos matemáticos.

El reconocimiento, medición, trazo y otras actividades afines al estudio de las figuras geométricas presentes en las fotografías de los diseños de los trajes regionales, desarrolla el pensamiento geométrico que abarca procesos de abstracción, área difícil de superar por el estudiante; genera la búsqueda de soluciones a planteamientos matemáticos, desarrolla la capacidad de análisis y el ambiente educativo se vuelve interesante, ameno y cooperativo entre estudiantes y docente. La auto investigación es otra de las actividades realizadas, para responder a los cuestionamientos y las estrategias para calcular el perímetro y área de las mismas fueron establecidas por el estudiante a través de las transformaciones de las figuras, descubriendo semejanzas en los cuadriláteros.

Cabe resaltar que la manipulación del recurso didáctico concreto, logra la contextualización de conceptos geométricos, porque forma parte del entorno de los estudiantes e incide positivamente en el análisis de las situaciones que se plantean al estudiante; como quedó demostrado al establecer los estudiantes relaciones entre figuras, para desarrollar una estrategia más simple en el cálculo de áreas y perímetros. Las actividades desarrolladas en la secuencia didáctica destacaron diversidad en la resolución de problemas matemáticos, sin aplicar fórmulas memorizadas.

La utilización de fotografías de trajes mayas en la evaluación diagnóstica y final como recurso didáctico, diversifica y cambia totalmente la modalidad del proceso evaluativo en matemática, proporcionando información valiosa acerca de los conceptos geométricos que posee el estudiante, ya que no causa bloqueo cognitivo y los motiva a responder con libertad; con esta modalidad las pruebas objetivas pasarían a un segundo plano; además se logra el desarrollo de habilidades y competencias matemáticas.
La diferencia entre lo observado por los estudiantes en la actividad diagnóstica y la actividad de evaluación final, es muy marcada y puede verificarse un aprendizaje significativo, lo cual hace pensar que se agotaron las fases de las situaciones didácticas que menciona Salinas (2010).

Algunas preguntas que quedan después de realizar el estudio se presentan a continuación, con la intención de que alguien interesado en el tema puede retomarlas en estudios futuros: ¿Qué conocimiento matemático tienen las tejedoras de huipiles?, ¿cómo se definen las formas, colores y patrones que siguen las tejedoras en la elaboración de huipiles?, ¿las formas geométricas que ven los profesores de matemática corresponden a las formas geométricas que construyen las tejedoras?, ¿qué resultados se consiguen al utilizar los huipiles como recurso didáctico con grupos de estudiantes de otros departamentos del país?, ¿qué opiniones emiten los docentes de matemática sobre el uso de material didáctico concreto para la enseñanza de la geometría? Estas y otras preguntas evidencian que hay mucho por estudiar sobre el tema.

\section{CONCLUSIONES}

- Los trajes típicos de Santa Catarina Ixtahuacán, son un recurso inédito e innovador en la presentación de conceptos matemáticos y una propuesta para la enseñanza de la geometría, siendo una fuente de motivación para el autoaprendizaje e investigación de conceptos geométricos.

- La manipulación de fotografías con diseños de trajes regionales, introduce al estudiante de manera natural e intuitiva al estudio de la geometría, asumiendo un papel activo en la construcción significativa de conceptos geométricos y en la medición de la superficie y perímetro de figuras planas, con estrategias propuestas por el estudiante y sin recurrir a la memorización de fórmulas.

- Los materiales didácticos en la enseñanza de la geometría, son útiles en el desarrollo de procesos cognitivos en el estudiante y facilita la abstracción de conceptos geométricos, potenciando así el pensamiento geométrico; además, favorece el aspecto didáctico del docente y sienta las bases para un aprendizaje sólido posterior. 
- Considerar la enseñanza de la geometría desde una perspectiva cultural, crea un ambiente de interés, motivación, capacidad de análisis y síntesis en los estudiantes, porque se toma como recurso elementos que forman parte de su cultura.

- El desarrollo de secuencias didácticas en la enseñanza de la Matemática, desarrolla y permite la manifestación de capacidades de los estudiantes y la formación del pensamiento matemático, porque establece relaciones y patrones matemáticos y la construcción del conocimiento, con el auxilio de materiales concretos.

- La resolución de problemas contextualizados que se presentan en la secuencia didáctica propicia la reflexión y análisis por medio de la experiencia, considerando como estrategia la resolución de problemas de su entorno para convertirse en un verdadero desafío y aprendizaje significativo.

- La modalidad de evaluación aplicada y las respuestas dadas por los estudiantes rompe el esquema tradicional con el que habitualmente se evalúan y que inhibe la participación espontánea y libre; el uso de recurso didáctico motiva la aplicación de los conocimientos adquiridos para la resolución de situaciones que se le presenta.

\section{REFERENCIAS}

Bronzina, L., Chemello, G., \& Agrasar, M. (2009). Aportes para la enseñanza de la Matemática. Santiago, Chile: Laborato rio Latinoamericano de Evaluación de la Calidad de la Educación.

Casasbuenas, C., Cifuentes, V., \& Ortega de N., M. (1998). Están dares Basicos de Competencias en Matemáticas. En M. d. Nacional, Matemáticas. Lineamientos Curriculares (págs. 47-95). Bogotá: Universidad Pedagógica Nacional.

Chacón, D., Trejos, A., Vargas, K., \& Venegas, A. (2005). Áreas sombreadas en Secundaria. Una propuesta para su Ense ñanza. Proyecto de Investigación y Formación Continua en la Enseñanza de la Matemática, 1-19.

Crespo, C. R. (2005). La Geometría en el Arte: Los Vitrales de las Catedrales Góticas. Acta Latinoamericana de Matemática Educativa, 313-320.

D’Amore, B. (2008). Epistemología, Didáctica de la Matemática y Prácticas de Enseñanza. Revista de la ASOVEMAT (Asocia ción Venezolana de Educación Matemática), 17 (1): 87-106. Diaz B., A. (2013). Guía para la Elaboración de una Secuen cia Didáctica. Comunidad de Conocimiento UNAM, 1-15.

Diaz t., N. D., \& Escobar M., S. V. (2006). Articulación de Activida des Didácticas con algunos aspectos Históricos de la Cul- tura y Matemática Maya en el Desarrollo del Pensamiento Espacial y Sistemas Geométricos del grado séptimo. San Juan de Pasto: Universidad de Nariño.

Flores, P., Lupiañez, J., Berenguer, L., Marín, A., \& Molina, M. (2011). Materiales y Recursos en el aula de Matemáticas. Granada: Departamento de Didáctica de la Matemática, Universidad de Granada.

Fuentes, C. (2017). La Fotografía, una Forma de Contextualizar la Matemática con Estudiantes del nivel medio. PIENSALO, 25-26.

Godino, J. D., \& Ruíz, F. (2002). Geometría y su didáctica para Maestros. Granada: Proyecto Edumat-Maestros. González, J. D. (2014). Comprensión de los Conceptos de Perímetro y Área y la Independencia de sus medidas, en el contexto de la agricultura del café. Medellín: Universidad de Antio quía.

Mántica, A. M., del Maso, M. S., Götte, M., \& Marzioni, A. (2002). La confusión entre área y perímetro. análisis de una pro puesta áulica. educación Matemática, 111-119.

Marmolejo Avenia, G. A., \& Gonzáles Astudillo,. M. (2015). El área de superficies planas en el campo de la educación matemática. Estado de la cuestión. Revista Electrónica de Investigación en Educación en Ciencias, 45-57.

Men. (2004). Pensamiento Geométrico y Tecnologías Compu tacionales. Bogotá, D.C., Colombia: Dirección de Calidad de la Educación Preescolar, Básica y Media. Ministerio de Educación Nacional.

MINEDUC. (2006). Prueba de Matemáticas Tercero Básico. Gua temala: DIGEDUCA.

Morales A., L. (s.f.). Consultoria de Etnomatemática. Guatemala: DIGEBI Ministerio de Educación.

Morales Aldana, L. (s.f.). Consultoria de Etnomatemática. Guatemala: DIGEBI Ministerio de Educación.

Peralta M., T., \& Murillo Ch., M. (2009). Elementos Teórico-Prác ticos para la Formación de Docentes de Educación Pri maria o Básica en Geometría y su Didáctica. San José, C. R.: Coordinación Educativa y Cultural Centroamericana.

Roldan O., G. J., \& Réndon R., H. d. (2014). Estrategia para el Esu dio del Área y el Perímetro de Figuras Planas articulada al modelo Socio Crítico para los estudiantes de la Institu ción Educativa María de los Ángeles Cano Márquez (Tesis de Maestría no Publicada). Medellín: Departamento de Ciencias Básicas, Universidad de Medellín.

Salinas M., M. E. (2010). Iniciación al Estudio de la Teoría de las Situaciones Didácticas. REVISTA Educación, Comunica ción y Tecnología, vol 5 No. 9 pág. 1-7.

Vargas V., G. (2013). El Modelo de Van Hiele y la Enseñanza de la Geometría. UNICIENCIA, Vol. 27, No. 1, pág 74-94. Villarroel, S., \& Sgreccia, N. (2011). Materiales Didácticos concretos en Geometría en primer año de Secundaria. NUMEROS, 78: 73-94. 


\section{Sobre los autores}

\section{Vilma Isabel Pérez Hernández}

Es Profesora de Matemáticas de la Escuela Normal Bilingüe Intercultural K'iche', Cantel, Quetzaltenango (desde hace 16 años), Licenciada en Educación de la Matemática y la Física, Maestría en Didáctica de la Matemática del Centro Universitario de Occidente, Quetzaltenango, Participación en la Reunión Latinoamericana de Matemática Educativa, miembro del Comité organizador de RELME 34, línea de investigación principal: Etnomatemática.

\section{Carlos Amilcar Fuentes Fuentes}

Es Editor en jefe de la Revista Fuentes de Aprendizaje en Innovación, Profesor Investigador del Centro Universitario de Occidente (desde hace 11 años), Licenciado en Educación de la Matemática y la Física, Maestro en Ciencias en Matemática Educativa del Instituto Politécnico Nacional de la Ciudad de México. Presidente de la Red de Formadores e investigadores de Matemática Educativa de Guatemala, Miembro del Comité Latinoamericano de Matemática Educativa, Coordinador del programa de maestría en didáctica de la matemática del CUNOC. Autor de libros de texto, artículos y ensayos.

Copyright (c) 2019 Vilma Isabel Pérez Hernández y Carlos Amilcar Fuentes Fuentes

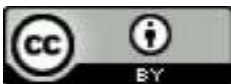

Este texto está protegido por una licencia Creative Commons 4.0.

Usted es libre para Compartir - copiar y redistribuir el material en cualquier medio o formato - y Adaptar eldocumento - remezclar, transformar y crear a partir del material- para cualquier propósito, incluso comercialmente, siempre que cumpla la condición de:

Atribución: Usted debe reconocer el crédito de una obra de manera adecuada, proporcionar un enlace a la licencia,e indicar si se han realizado cambios. Puede hacerlo en cualquier forma razonable, pero no de forma tal que sugieraque tiene el apoyo del licenciante o lo recibe por el uso que hace. 